\title{
Rate-dependent tipping and early warning in a thermoacoustic system under extreme operating environment
}

\author{
Xiaoyu Zhang ${ }^{\mathrm{a}}$, Yong Xu ${ }^{\mathrm{a}, \mathrm{b}, *}$, Qi Liu ${ }^{\mathrm{a}}$, Jürgen Kurths ${ }^{\mathrm{c}}$, Celso Grebogid ${ }^{\mathrm{d}}$ \\ ${ }^{a}$ School of Mathematics and Statistics, Northwestern Polytechnical University, Xi'an, 710072, China \\ ${ }^{b}$ MIIT Key Laboratory of Dynamics and Control of Complex Systems, Northwestern Polytechnical \\ University, Xi'an, 710072, China \\ ${ }^{c}$ Potsdam Institute for Climate Impact Research, Potsdam 14412, Germany \\ ${ }^{d}$ Institute for Complex Systems and Mathematical Biology, School of Natural and Computing Sciences, \\ King's College, University of Aberdeen, Aberdeen AB24 3UE, United Kingdom
}

\begin{abstract}
Thermoacoustic instability has been an important challenge in the development of highperformance combustion systems, as it can have catastrophic consequences. The process of a sudden change in the dynamical behaviour of a thermoacoustic system from a low to a high amplitude thermoacoustic instability, actually entails as a tipping point phenomenon. It has been found that when rate-dependent parameters are considered, a tipping-delay phenomenon may arise, which helps in the control of undesirable states that give rise to thermoacoustic instabilities. This work aims at understanding rate-dependent tipping dynamics of the thermoacoustic system with both time-varying parameters and a non-Gaussian Lévy noise. The latter better describes the severe operating environment of such systems than simpler types of noise. Through numerical simulations, the tipping dynamical behaviour is analysed by considering the rate-dependent parameters coupled with the main parameters of the Lévy noise, including the stability and skewness indices, and the noise intensity. In addition, we investigate the effectiveness of early warning indicators in rate-dependent systems under Lévy noise excitation, and uncover a relationship between warning measures and the rate of change in the parameters. These results inform and enlighten the development and design of power combustion devices, and also provide researchers and engineers with effective ideas to control thermoacoustic instability and the associated tipping dynamics.
\end{abstract}

Keywords Thermoacoustic instability; Lévy noise; Rate-dependent tipping; Early warning

\footnotetext{
${ }^{*}$ Corresponding author

Email addresses: xiao_yu_zhang@yahoo.com (Xiaoyu Zhang), hsux3@nwpu.edu.cn (Yong Xu), liuqi1780280327@yahoo.com (Qi Liu), Juergen.Kurths@pik-potsdam.de (Jürgen Kurths), grebogi@abdn.ac.uk (Celso Grebogi)
} 


\section{Leading paragraph}

Thermoacoustic systems are closely related to propulsion combustion devices such as solid and liquid rocket motors, aero engines, and gas turbines. A thermoacoustic instability arises when there is a positive feedback between the non-constant exothermic rate in the combustion chamber and the acoustic field. Thermoacoustic instabilities lead to self-excited large amplitude pressure oscillations in the combustion chamber. These typically undesirable pressure oscillations can lead to catastrophic consequences such as structural damage due to excessive heat transfer and vibration, ballistic anomalies in the engine, damage to electronic equipment in aircrafts and satellites, and even to rocket launch missions due to engine disintegration. Therefore, the occurrence of thermoacoustic instability has been a major problem faced during the development of high-performance combustion systems. So far, studies of the thermoacoustic instability are limited to the excitation of Gaussian noise, which has limitations in describing large jumps. However, the effect of extreme severe operating environments on thermoacoustic instability cannot be ignored. So it is crucial to introduce a more appropriate noise portrayal in the study: non-Gaussian Lévy noise. Considering that the control parameters in real industrial thermoacoustic systems are time-varying, this work provides referenceable control strategies and early warning signals for thermoacoustic instability avoidance based on the rate-dependent mathematical model of thermoacoustic systems.

\section{Introduction}

The understanding of thermoacoustics dates back more than two hundred years, when Higgins [1] first discovered the thermoacoustic effect. When he placed a hydrogen flame in the proper position in a vertical tube with open ends, a sound was excited in the tube, a phenomenon that has been called a "singing flame". In 1859, Rijke [2] developed further the Higgins tube, the Rijke tube with its rich accompaniment and bright sound, which became the simplest example for studying thermoacoustic instability [2, 3]. Since then, researchers have continued to develop and refine thermoacoustic theory based on their predecessors. The understanding and studying of thermoacoustic instability is a challenging problem, since they are the result of complex interactions between acoustics, combustion, and hydrodynamics, and it is an urgent problem due to the important device applications.

Traditionally, the transition to thermoacoustic instability is described as a Hopf bifurcation, in which the dynamics of the system undergoes an abrupt change from a combustion noise state to a thermoacoustic instability [4]. This is actually a tipping phenomenon that occurs in the thermoacoustic systems. Tipping is an event in a multi-stable system where a 
very small change in inputs causes a sudden, disproportionate change in outputs [5]. From a mathematical perspective, Ashwin and his co-workers [6] classified tipping into the following three categories based on the intrinsic occurrence mechanism of tipping, namely bifurcationinduced tipping (B-tipping), noise-induced tipping (N-tipping), and rate-induced tipping (R-tipping). Specifically, B-tipping refers to the bifurcation behaviour of the system when the value of external forces or internal parameters of the system slowly exceeds a critical threshold. The saddle-node and Hopf bifurcations are typical B-tipping. There are many natural examples of B-tipping; for example, the dieback of the Amazon rainforest is a typical B-tipping phenomenon [7]. N-tipping is a phenomenon in which the system state changes after a noise disturbance. The most typical example of the N-tipping phenomenon in climate science is the Dansgaard-Oeschger event [8]. R-tipping is a new concept introduced only in recent years, which considers a system in which the parameters no longer remain stationary, but become a new time-dependent variable in the system. Tipping occurs when the rate of change of a system parameter exceeds a certain critical value. In fact, R-tipping describes a phenomenon in which the system parameters change too quickly for the system to adapt. A recent paper provides a R-tipping example that describes the collapse mechanism of the Atlantic meridional flip-flop [9]. Here the Atlantic Meridional Overturning Circulation (AMOC) is an important component of the global ocean circulation, and when the rate of ice melting increases beyond a certain critical rate of change, it leads to the collapse of the AMOC. Since the AMOC plays an important role in world's climate and in suppressing global warming, its collapse could lead to irreversible climate change. It is, therefore, of great interest to conduct research on R-tipping.

Consider that in most industrial thermoacoustic systems, the control parameters are varied over time to meet the changing demands. Therefore, it is necessary for us to consider R-tipping in a thermoacoustic system. In particular, when a rate is introduced into the system, the dynamics hovers around the previous stabilization point due to inertia, i.e., the system undergoes a tipping-delay phenomenon. Tipping-delay phenomenon refers to the delay in the value of the parameter where the rate makes tipping occur as compared to the proposed steady-state case. This tipping-delay phenomenon may provide new control strategies for thermoacoustic instability avoidance, which also reflects the importance of conducting research on R-tipping in thermoacoustic systems.

There have been many studies on R-tipping in thermoacoustic systems. Bonciolini et al. [10] experimentally and numerically verified the tipping-delay phenomenon in rate-dependent thermoacoustic systems driven by Gaussian white noise. Subsequently, they also found that 
thermoacoustic instabilities can be circumvented when the rate of parameter change is fast enough [11]. Further, Zhang et al. [12, 13] considered the correlation time of noise, extended the above findings to systems excited by Gaussian colored noise, and obtained the effect of noise correlation time coupled with the rate of parameter change on thermoacoustic systems.

In most of the above studies, the random noise is usually assumed to be Gaussian noise for simplicity of treatment. Gaussian noise is a formal derivative of Brownian motion and is generally used to describe continuous small random perturbations. Gaussian noise has been widely used in many fields because it satisfies the central limit theorem and is relatively simple to handle and easy to derive the theory. However, Gaussian noise is actually an ideal noise source, which portrays the normal diffusion situation and can only describe small fluctuations close to the average value, and cannot describe large rises and jumps. There is strong evidence in practice that many complex phenomena in nature are perturbed by nonGaussian noise, such as transient noise in electric currents [14], random energy fluctuations in statistical mechanics [15], and stock price variations in option pricing [16]. The non-Gaussian stochastic processes corresponding to these non-Gaussian noises have a path band jump discontinuity and a probability density function (PDF) showing a heavy-tailed distribution. Lévy noise is a typical class of non-Gaussian noise that can be used to describe systems where continuous and jumping random factors take action together, and has been well used in many practical systems such as the millennium climate change system [17, 18], laser gyroscopes [19], and seismic environments [20]. Considering that propulsion devices such as rocket engines, which are closely related to thermoacoustic systems, are often in extreme operating environments such as extreme cold and heat, and may in turn be subject to extreme loads or extreme excitations. Such random influences with occasional jumps are more suitable to be described by Lévy noise which is more common than Gaussian noise. Therefore, in this work, we consider the dynamical behaviours of a thermoacoustic system driven by a Lévy process.

Given that the changes caused by tipping are to some extent irreversible and can have significant adverse consequences for the system, predicting the point at which tipping will occur before it happens is a critical topic. In general, such predictions are very difficult because the system state may barely change until reaching a critical point. However, in recent years, some results have been achieved in proposing effective early warning signals in systems with B-tipping. Based on the critical slowing down theory, the lag-1 autocorrelation and the variance become the two most commonly used early warning signals [21]. In addition, effective early warning indicators are also proposed in the N-tipping system by combining 
the concept of basin stability [22, 23]. The study of early warning signals is more difficult in systems where both rate and noise are considered, because the parameters are constantly changing making the signals difficult to identify. For rate-dependent thermoacoustic systems, a preliminary exploration of early warning for systems excited by Gaussian white noise was made by Pavithran et al [24]. Nevertheless, it remains to be verified whether the classical early warning indicators are still effective in rate-dependent systems when the noise excitation becomes a more general Lévy noise with jumps. This work will attempt to provide answers to these questions.

This work focuses on the dynamical transition behaviours of a thermoacoustic system under non-Gaussian Lévy noise excitation, in which when the rate of parameter change is also considered. The influences of the Lévy noise parameter on the tipping behavior, especially on the tipping-delay phenomenon specific to rate-dependent systems, are investigated by numerical simulations. Further, the tipping behaviour under the coupling of the parameter change rate with the Lévy noise parameters are analysed in details. In addition, we verify the effectiveness of early warning signals in rate-dependent thermoacoustic systems and give the relationship between the warning effect and the rate of parameter change.

The main structure of this paper is as follows. The second section briefly introduces the classical mathematical models in thermoacoustic systems and the basics of Lévy noise. The third section gives the main findings of this work in three parts. The fourth section makes some summaries of this paper.

\section{Thermoacoustic system with Lévy noise}

In recent years, with the deepening of research, the dynamical behaviour of thermoacoustic system is better expressed by a Helmholtz equation [10], that is

$$
\frac{\partial^{2} x}{\partial t^{2}}-c^{2} \nabla^{2} x=(\gamma-1) \frac{\partial q}{\partial t}
$$

where $x, c, \gamma$ and $q$ denote the acoustic pressure, the speed of sound, the heat capacity ratio, and the fluctuating component of the heat release rate, respectively. The simplified mathematical model (2.2) of the thermoacoustic system is obtained through Laplace transform, orthogonal projection, and truncated Taylor expansion approximation of the nonlinear term $[11]$

$$
\ddot{x}-\left(v+\beta_{1} x^{2}-\beta_{2} x^{4}\right) \dot{x}+\omega_{0}^{2} x+\beta_{0} x^{3}=\xi(t),
$$

where $v$ is related to the linear growth rate, and $\beta_{0}, \beta_{1}$, and $\beta_{2}$ are real parameters. Here $\xi(t)$ is Lévy noise, which is the formal derivative of Lévy process with respect to time $t$. 
Lévy process $L=\left\{L_{t}, t \geq 0\right\}$ is a stochastic process which has independent and stationary increments and $L_{0}=0$ almost surely. In addition, $L$ is stochastically continuous, i.e, for all $a>0$ and for all $s \geq 0, \lim _{t \rightarrow s} \mathbb{P}\left(\left|L_{t}-L_{s}\right|>a\right)=0$, where $\mathbb{P}$ is the probability measure in the probability space $(\Omega, \mathcal{F}, \mathbb{P})$ of Lévy process. Lévy process can be described by its characteristic function

$$
\phi(t)=\left\{\begin{array}{l}
\exp \left\{i \mu t-\sigma^{\alpha}|t|^{\alpha}\left[1-i \beta \operatorname{sgn}(t) \tan \left(\frac{\pi \alpha}{2}\right)\right]\right\}, \quad \alpha \in(0,1) \cup(1,2], \\
\exp \left\{i \mu t-\sigma|t|\left[1+i \beta \operatorname{sgn}(t) \frac{2}{\pi} \ln |t|\right]\right\}, \quad \alpha=1,
\end{array}\right.
$$

where $\alpha \in(0,2]$ is the stability index, $\beta \in[-1,1]$ is the skewness index, and $\sigma>0$ represents the scale parameter $\sigma=D^{1 / \alpha}$ and $D$ is the noise intensity.
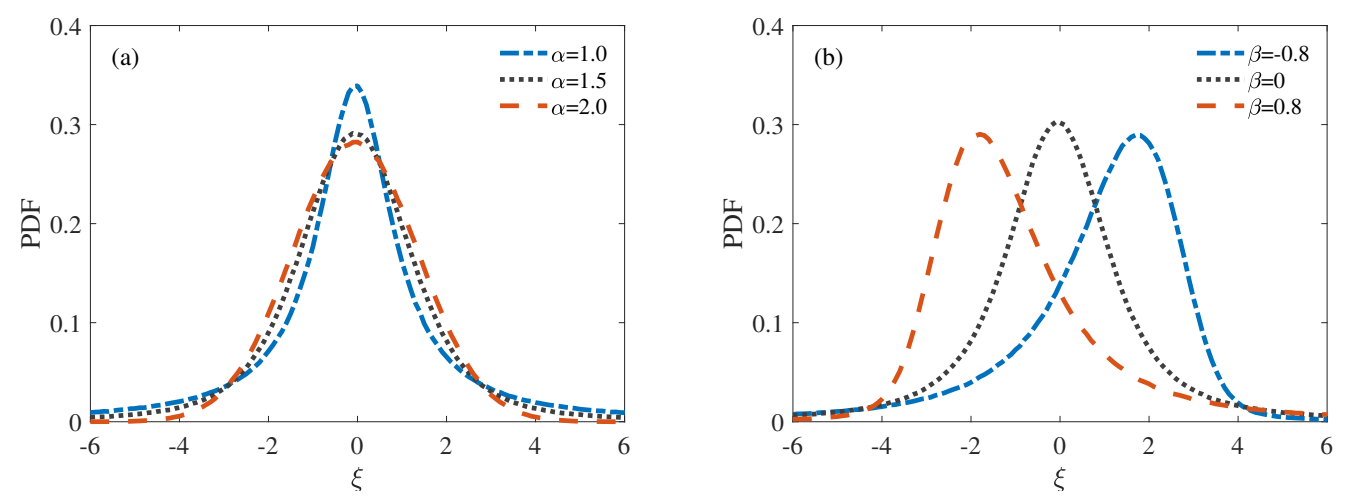

Figure 1: The probability density functions (PDFs) of Lévy noise. (a) Results under different stability index $\alpha$ with $\beta=0, D=1$. (b) Results under different skewness index $\beta$ with $\alpha=1.3, D=1$.

Figure 1 exhibits the PDFs of Lévy noise. As can be seen from Figure 1(a), the smaller $\alpha$ makes the distribution narrower and higher, and it has a heavy tail. When $\alpha=2$, it degenerates to Gaussian distribution. Figure 1(b) shows that the PDFs are symmetric when $\beta=0$. Under the condition of $1<\alpha<2$, for $\beta>0$, the PDF is skewed to the left, while $\mathrm{PDF}$ is skewed to the right for $\beta<0$. Since the moments above the $\alpha$ order diverge [25], the numerical simulation is prone to overflow when $\alpha \leq 1$. To ensure that the results are theoretically meaningful, the following research of this paper is focused on the case of $1<\alpha \leq 2$ to study influences of the stability index, the skewness index and the noise intensity of the Lévy noise on the thermoacoustic system (2.1).

\section{Results}

\subsection{Effect of Lévy parameters on rate-dependent systems}

In this section, we mainly study the influences of several important parameters of Lévy noise on the rate-dependent thermoacoustic system (2.2), especially the tipping-delay phenomenon. The form of parameter variation considered in this section is a linearly increasing 
function, i.e. $v(t)=v_{0}+R t$, where $v_{0}$ is the initial value and $R$ is the rate of the system parameters.

\subsubsection{Stability index $\alpha$}

We first consider the stability index $\alpha$. When $\alpha=2$, the Fokker-Planck-Kolmogorov (FPK) equation of the amplitude can be deduced by a stochastic averaging method. Furthermore, the approximate analytical solution of the probability density can be obtained by solving the FPK equation [12]. The contour diagram in Figure 2 describes the transient dynamical behavior of the system when $\alpha=2$, which is the case of Gaussian white noise. The blue line is the bifurcation behaviour in the steady state of the deterministic dynamics given by equation (2.2) without the noise term, in which the solid line indicates stability of the system and the dashed line indicates instability. The red line is the change rate of amplitude with time $d A / d t$, which is obtained after considering the mean path of amplitude $A(t)$ over multiple realizations due to the effect of randomness. We take the maximum of the change rate (i.e., the vertical purple line) as the time of tipping [24]. The bifurcation theory calculation gives that under steady state, tipping occurs at $t=0.45$, and the dynamics jumps from low-amplitude to high-amplitude state. But after the introduction of rate $\mathrm{R}$, the tipping occurs around $t=1$.1. Therefore, the phenomenon of rate-dependent tipping-delay from $t=0.45$ to $t \approx 1.1$ appears in the system, which has been systematically studied in $[12,13]$.

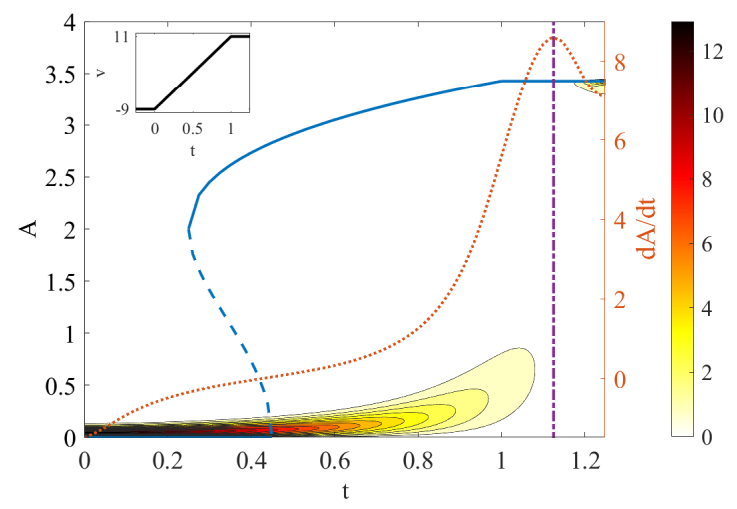

Figure 2: The transient dynamical behaviour of the system when $\alpha=2$, i.e, excited by Gaussian white noise. The internal diagram shows the form of the parameter changes $v(t)=v_{0}+R t$ for $v_{0}=-9, R=20$ in this section. Contour map represents the transient probability density, in which the darker the colour, the larger the probability. The blue line describes the bifurcation behaviour in the steady state of the deterministic dynamics for reference. The rate of change of amplitude is displayed by the red line to identify the onset of thermoacoustic instability. The maximum rate of change of $A$, considered to be the tipping time, is indicated by the vertical purple line at $t \approx 1.1$. Other parameters are $\beta_{1}=8, \beta_{2}=2$, and $\omega_{0}=120 \times 2 \pi$.

When the stability index $\alpha \neq 2$, the exact expression of PDF cannot be obtained, so the 
stochastic Runge-Kutta and Monte Carlo simulations for the equation (2.2) are implemented in our investigation. Figure 3 shows the amplitude $A$ for different $\alpha$ as a function of $t$. The red dotted line represents the high amplitude steady state of the system. It can be seen from the Figure 3 that when $\alpha$ is small, the noisy dynamics crosses the high amplitude state intermittently, and the smaller $\alpha$ is, the higher the jump frequency is. These intermittent jumps are similar to the extreme events, which indicates that the model can be used to describe the influence of extreme working environment on the thermoacoustic system. With the increase of $\alpha$, the dynamics tends to Gaussian white noise, and the system is stable, reaching high-amplitude state from low-amplitude one, after some time.
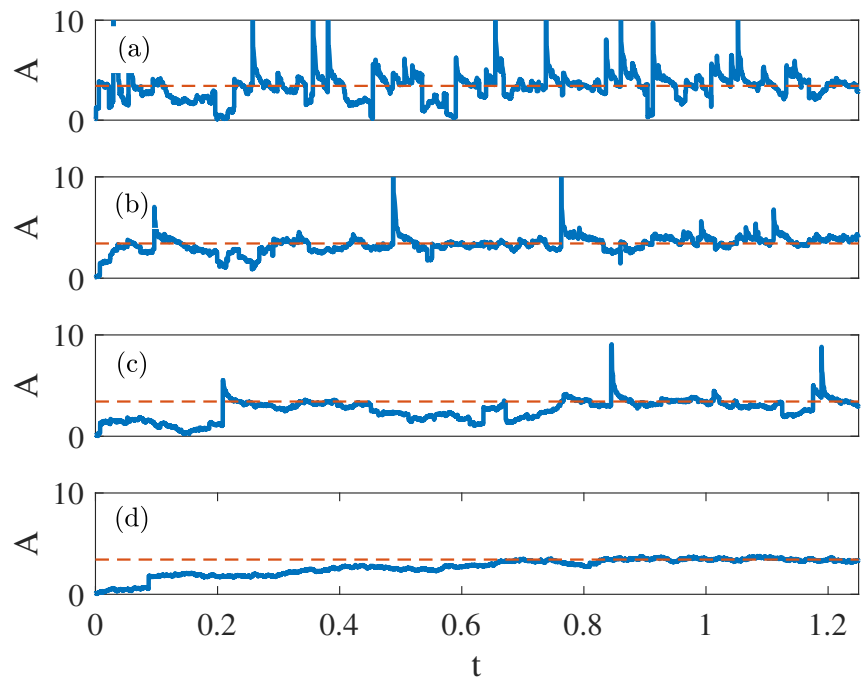

Figure 3: The amplitude $A$ under different $\alpha$. (a) $\alpha=1.1$, (b) $\alpha=1.3$, (c) $\alpha=1.5$, (d) $\alpha=1.9$. Other parameters are $\beta=0, D=10^{5}$.

Next, we look at the transient dynamical behaviour of the rate-dependent system excited by Lévy noise with different $\alpha$. As shown in Figure 4, tipping occurs fasterly with the decrease of $\alpha$, which corresponds to the result in Figure 3. When $\alpha$ is small, the intermittent jumping of the trajectory makes tipping easier. This shows that compared with Gaussian noise, Lévy noise makes particles jump to the high amplitude state of the system earlier. That is, Lévy noise makes the thermoacoustic system to enter the thermoacoustic instability state more quickly.

We use the timing index of tipping, that is, the maximum value of amplitude change rate $d A(t) / d t$ mentioned in Figure 2, to quantify the tipping-delay phenomenon under different $\alpha$. It can be seen from Figure 5(a) that the larger $\alpha$ is, the later the tipping occurs. The grey dashed line is the occurrence time of tipping in the steady state, and the difference between 

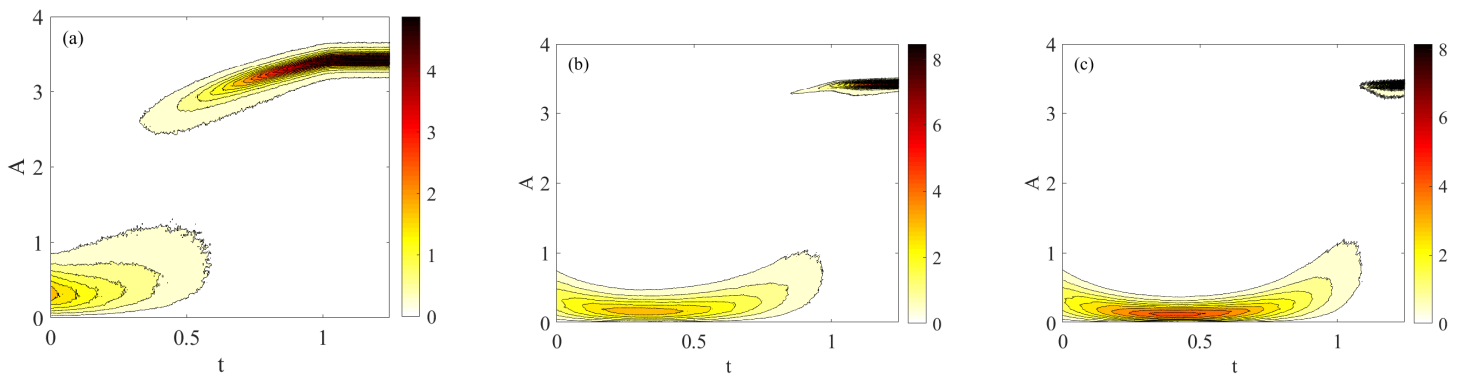

Figure 4: The contour plot represents the PDF of amplitude $A$ under different stability index $\alpha$. (a) $\alpha=1.1$, (b) $\alpha=1.5$, (c) $\alpha=1.9$. Other parameters are $\beta=0, D=10^{5}$.

it and the highest point of amplitude change rate is the delay time $\delta$ of the tipping-delay phenomenon. We find that although compared with Gaussian noise, Lévy noise promotes the timing of tipping, including the tipping-delay phenomenon. Specifically, the relationship between the delay time $\delta$ and $\alpha$ is shown in Figure 5(b). The delay time $\delta$ increases with the increase of $\alpha$, and the increasing speed slows down with the increase of $\alpha$, gradually tending to be flat. This can be used to reduce the thermoacoustic instability by increasing $\alpha$, and the effect of control is significant when $\alpha$ is small.
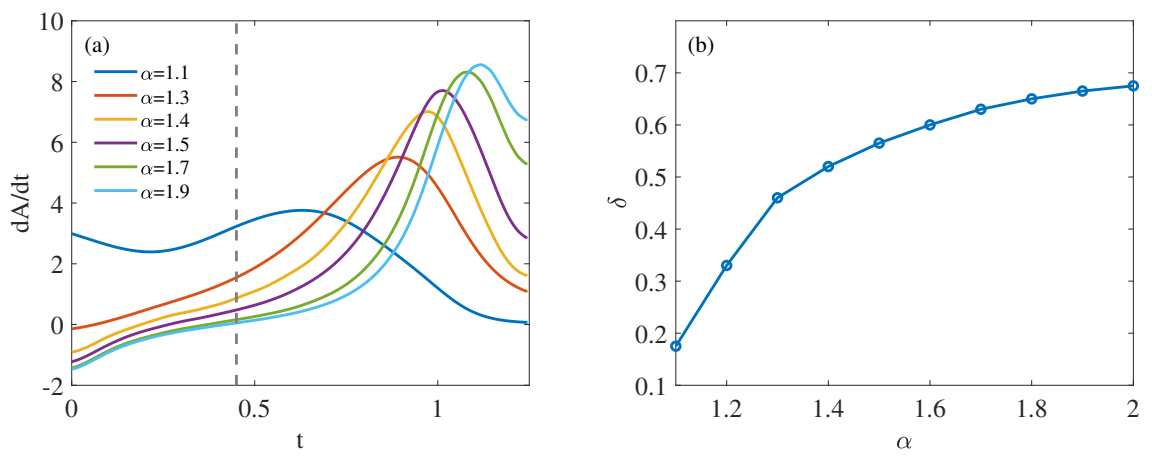

Figure 5: (a) Timing index of tipping, given by $d A(t) / d t$, under different stability index $\alpha$. (b) Variation of delay time $\delta$ of tipping-delay phenomenon with different stability index $\alpha$.

\subsubsection{Skewness index $\beta$}

Next, we consider the influence of the skewness index $\beta$ on the rate-dependent thermoacoustic system. Firstly, we consider the paths of the amplitude $A$, as shown in Figure 6 for details. Here, take the values at both ends of the range $[-1,1]$ as an example. There are no significant differences in the shape, the size and frequency of intermittent jumps among the paths of the amplitude, and the results are similar under different $\alpha$. Therefore, we come to the conclusion that there is not much difference in the paths of the amplitude $A$ when $\beta$ is different. 



Figure 6: Paths of the amplitude $A$ under different skewness index $\beta$. (a) $\alpha=1.1, D=10^{5}$, (b) $\alpha=1.9$, $D=10^{5}$.

For the response $x$, from Figure 7(a), when the skewness index $\beta>0$, the large jump of the system appears more in the direction of $x>0$, while when $\beta<0$, the large jump of the system appears more in the $x<0$ direction. Furthermore, in order to show the effect of $\beta$ on the response, the probability density is calculated statistically, as shown in Figure 7(b). Among them, the blue solid line is the probability density for $\beta=1$, the red dotted line is the probability density for $\beta=-1$, the ordinate corresponds to $x \in[-10,10]$ of the left graph, and the abscissa takes the logarithm value of the PDF in order to more clearly show the change of the probability density. It can be seen from the Figure 7 that when $\beta=1$ the probability density shows a heavy tail in the direction of $x>0$, and when $\beta=-1$ the probability density is more concentrated in the direction of $x<0$. Moreover, the PDFs of $\beta=1$ and $\beta=-1$ are symmetric with respect to $x=0$, and the area of the blue and red shaded parts are equal. Therefore, the skewness index $\beta$ affects the response $x$, but has little effect on the amplitude $A$. The reason is that in the process of calculating amplitude $A$, the response $x$ is squared to eliminate the influence of $\beta$.
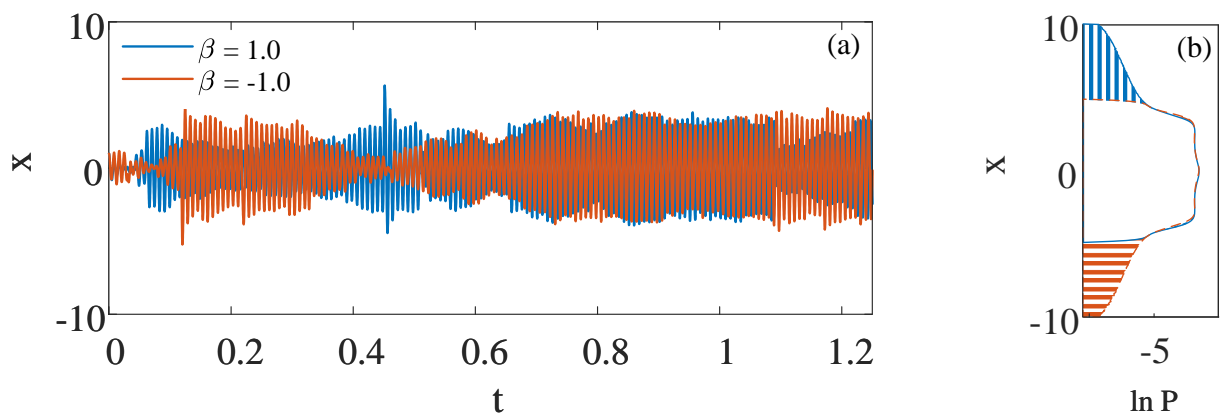

Figure 7: (a) The paths of the response $x$ under different skewness indices $\beta$. (b) Probability density of semi-logarithmic graph under different skewness indices $\beta$. 


\subsubsection{Noise intensity $D$}

Finally, we consider the effect of noise intensity $D$ on tipping. Figure 8 shows the transient probability density of the system under different noise intensities. It can be seen that the higher the noise intensity $D$ is, the earlier the tipping occurs.
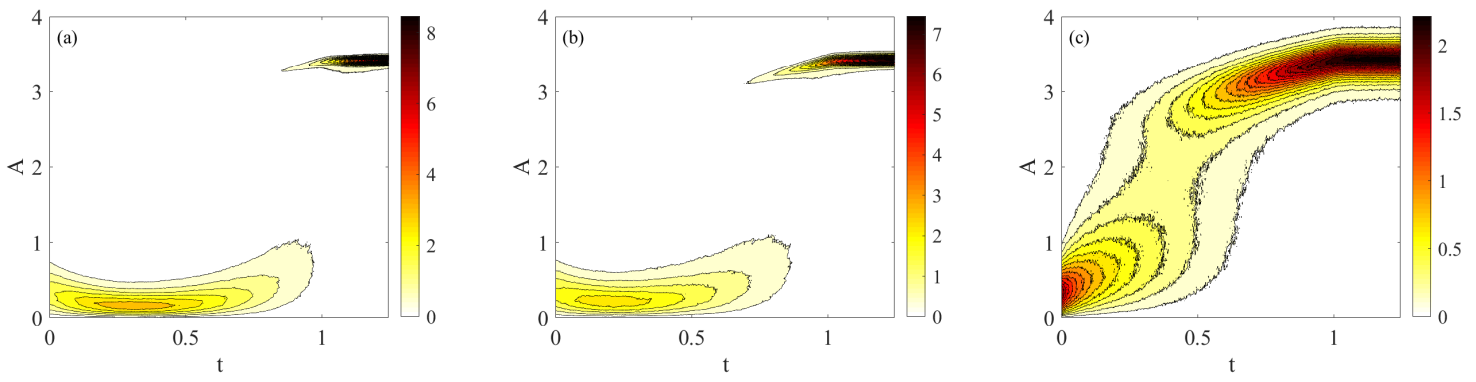

Figure 8: The contour plot represents the PDF of amplitude $A$ under different noise intensity $D$. (a) $D=5.0 \times 10^{3}$, (b) $D=10^{4}$, (c) $D=5.0 \times 10^{4}$. Other parameters are $\alpha=1.5, \beta=0$.

Similarly, we use the timing index of tipping to observe the tipping-delay phenomenon under different noise intensities. In Figure 9(a), the tipping time in the steady state, represented by the dashed line, is taken as a reference, and the tipping-delay phenomenon still exists when the noise intensity $D$ is changed. Combining with Figure $9(\mathrm{~b})$, it is found that the larger the noise intensity $D$ is, the shorter the delay time $\delta$ is, and the delay time is basically linear with the noise intensity. This reveals that the noise intensity of Lévy noise affects the thermoacoustic instability.
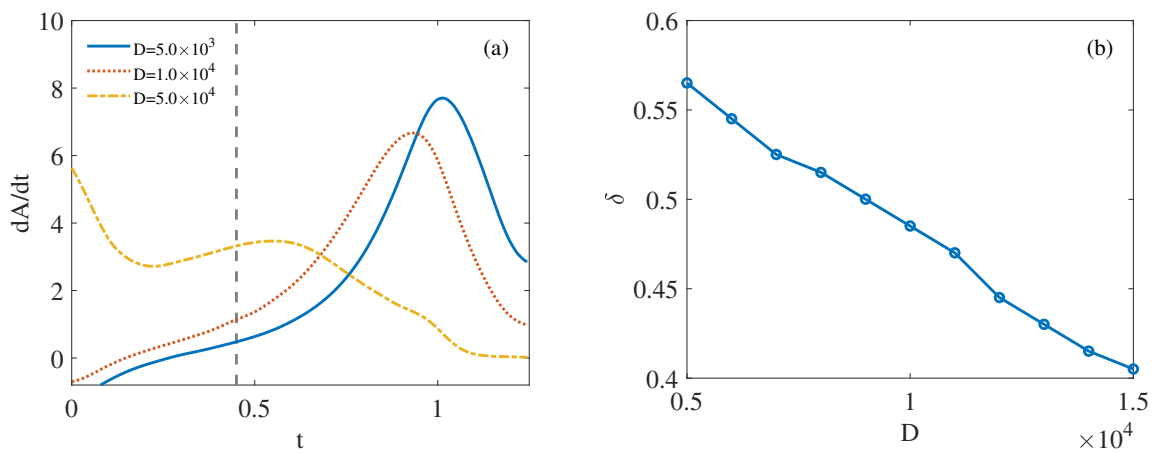

Figure 9: (a) Timing index of tipping under different noise intensity $D$. (b) Variation of delay time $\delta$ of tipping-delay phenomenon with different noise intensity $D$.

\subsection{Effect of rate coupled with Lévy parameters}

In this section, we mainly study the influence of the rate of the system parameters coupled with Lévy parameters on the rate-dependent thermoacoustic system. In view of the 
conclusion in the previous section that the skewness index has little effect on the amplitude, we consider only the stability index and the noise intensity in this section. The parameter time dependence is considered to be a linear function first increasing and then decreasing, as detailed in the diagram inside Figure 10(a). Moreover, we unify the time scale so that the systems with different rates can be considered in the same parameter space.

\subsubsection{Coupling of rate and stability index}
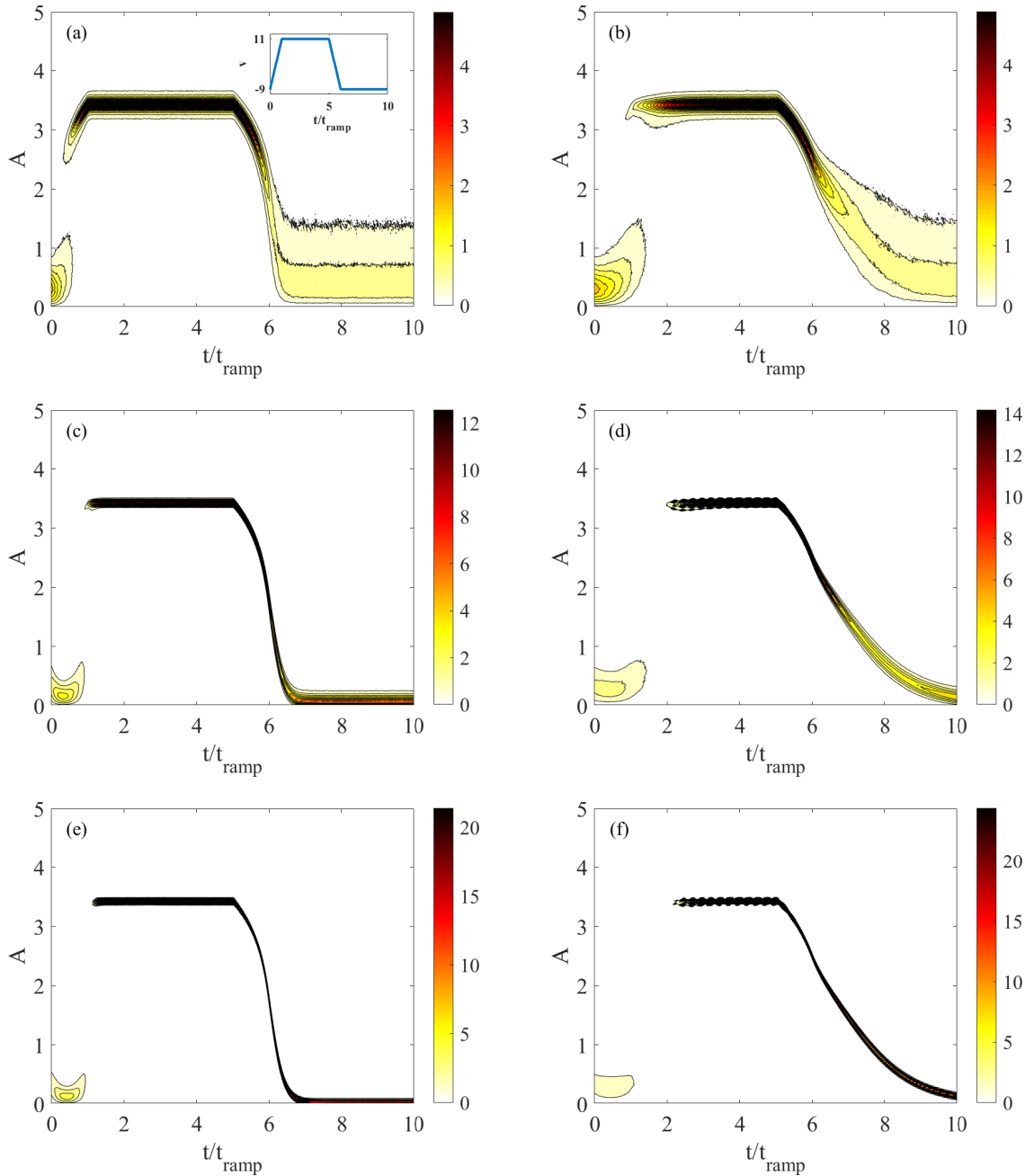

Figure 10: The contour plot represents the PDF of system amplitude $A$ under different stability index $\alpha$ and the rate of parameter $R$. The internal diagram in (a) shows the form of the parameter changes in this section. Here, $D=5.0 \times 10^{3}, \beta=0$. The stability index $\alpha$ increases from top to bottom. (a)(b) $\alpha=1.1$, (c)(d) $\alpha=1.5$, (e)(f) $\alpha=1.9$. Each column corresponds to the results for a particular rate. (a)(c)(e) $R=20$, (b)(d)(f) $R=100$.

Figure 10 shows the transient dynamical behaviour of the amplitude $A$ under different $\alpha$ and $R$ versus $t / t_{\text {ramp }}$, where $t_{\text {ramp }}=\left(v_{m}-v_{0}\right) / R=[11-(-9)] / R$ to normalize the time. Among them, the left column is the case of a small rate of the parameter change, and the 
right column is the case of a large rate, the value of $\alpha$ gradually increases from top to bottom. Consistent with the results in the previous section, with the increase of $\alpha$, the tipping-delay time becomes longer and the state of particles becomes more concentrated. On this basis, considering the effect of rate of change, it is found that the increase of rate makes the delay time longer. The contour map of tipping time under different $R$ and $\alpha$ is seen in Figure 11 when the parameter increases. That is, when the low amplitude state jumps to the high amplitude state, the time of tipping occurrence increases with increasing $R$ and $\alpha$, and the delay time also increases. This shows that both the rate of change $R$ and the stability index $\alpha$ can promote the tipping-delay phenomenon.

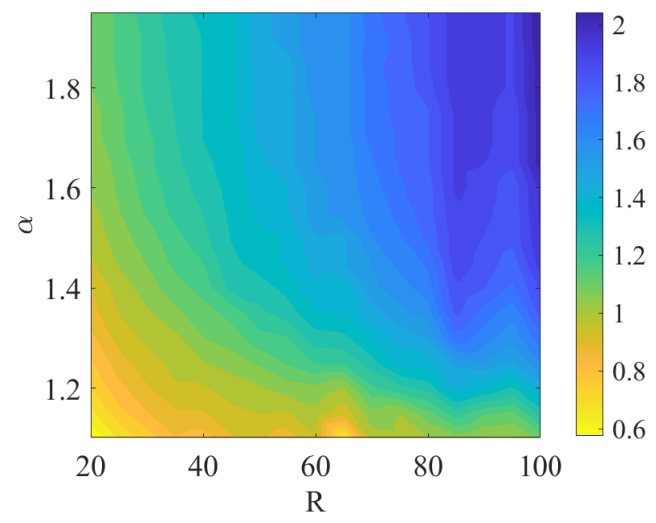

Figure 11: Contour map of the occurence time of tipping under different stability index $\alpha$ and the rate of parameter $R$.

\subsubsection{Coupling of rate and noise intensity}

Next, the influence of the noise intensity $D$ and rate $R$ on the rate-dependent thermoacoustic system is considered. It can be seen from Figure 12 that the large noise intensity $D$ makes the particle state more dispersed, which makes tipping to happen earlier. But large rate $R$ prolongs the occurrence time of tipping. Therefore, the occurrence time of tipping in Figure 12(a) with small noise intensity and small rate is similar to that in Figure 12(d) with larger noise intensity and larger rate, and Figure 13 shows this rule more clearly. Consequently, in addition to the control strategy mentioned in the previous section 3.1, we can also mitigate the thermoacoustic instability by increasing the rate of change of the parameters when the noise intensity is relatively high.

\subsubsection{The role of rate of parameter change}

The parameters considered in this section are first an increasing function and then a decreasing one. After unifying the time scale, the system with different rate is guaranteed to 

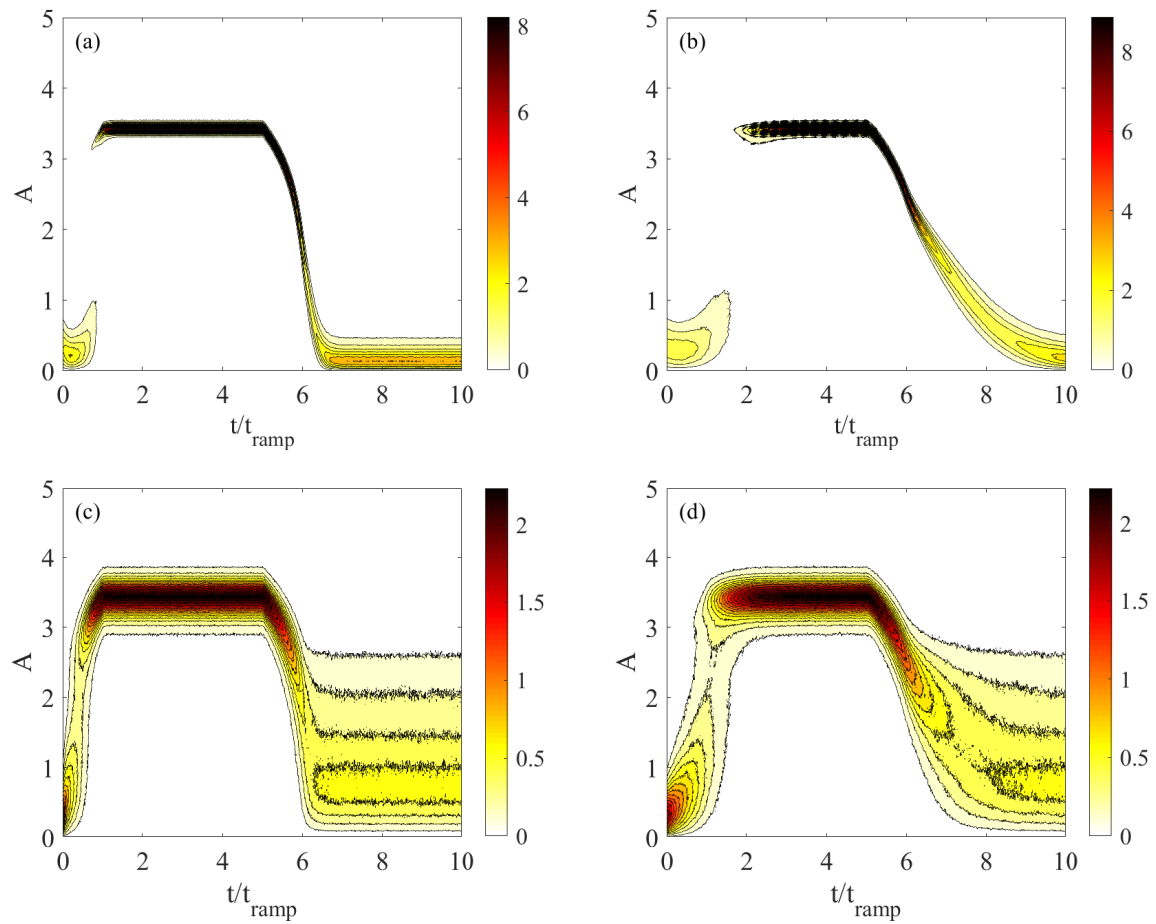

Figure 12: The contour plot represents the PDF of system amplitude $A$ under different noise intensity $D$ and the rate of parameter $R$. Here, $\alpha=1.5, \beta=0$. The noise intensity $D$ increases from top to bottom. (a)(b) $D=10^{4}$, (c)(d) $D=5.0 \times 10^{4}$. Each column corresponds to the results for a particular rate. (a)(c) $R=20,(\mathrm{~b})(\mathrm{d}) R=100$.

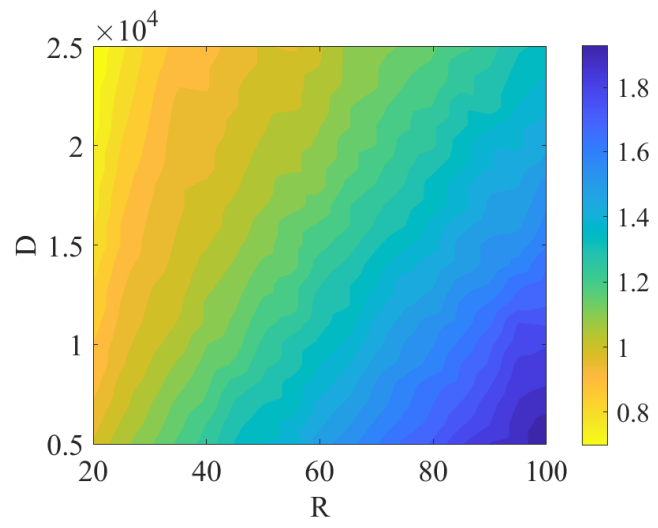

Figure 13: Contour map of the occurence time of tipping under different noise intensity $D$ and the rate of parameter $R$. 
be in the same parameter space. We find that the parameter value of tipping is delayed after introducing the rate of change. Furthermore, it can be seen from Figure 14 that through the timing index of tipping, it is found that when the parameter increases, the size of rate has an obvious influence on tipping-delay phenomenon, that is, the abscissa values of the maximum point of the two curves in the Figure 14 is obviously different. However, when the parameter falls, the abscissa values of the minimum points of the two curves are almost the same, which indicates that the size of rate of parameter change has little effect on the tippingdelay phenomenon. It is noticed that the effect of rate on the rise and fall of parameter is different, not only in the system excited by ordinary Lévy noise, but also in the case of $\alpha=2$ Gaussian white noise.
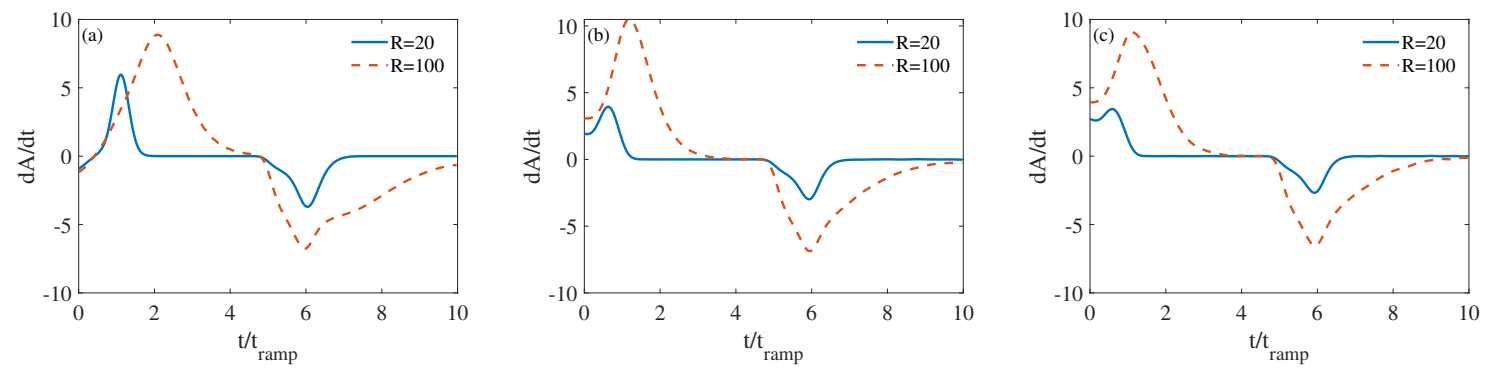

Figure 14: Timming index of tipping in the form of increasing first and then decreasing. (a) $\alpha=2, D=$ $5.0 \times 10^{3}$, (b) $\alpha=1.9, D=10^{5}$, (c) $\alpha=1.1, D=5.0 \times 10^{3}$.

In order to explore the causes of the above phenomena and the internal mechanism of tipping when the parameters rise and fall, we observe the change of the potential function. Taking the case of Gaussian white noise in Figure 14(a) as an example, the expression of the potential function can be derived from the stochastic differential equation obtained by the stochastic averaging method. It can be seen from Figure 15(a) that when the parameter increases, that is, $v$ increases from -9 to 11, the potential function changes from monostable state to bistable state and then into monostable state, while when the parameter decreases, the process is the opposite.

Combining with the change curve of barrier height in Figure 15(b), it can be concluded that the initial condition of parameter is $v=-9$, and the barrier height of potential function is about 60. The initial condition of parameter falling is $v=11$, and the barrier height of potential function is about 35. When the barrier height decreases close to 0, the system enters into bistability, and tipping is very easy to occur. The higher the barrier height is, the more difficult it is for tipping to occur. Therefore, for tipping, the difficulty is greater when the parameters rise than when they fall. At this time, rate, as a factor contributing to the occurrence of tipping, plays a more obvious role when it is more difficult. That is, if 

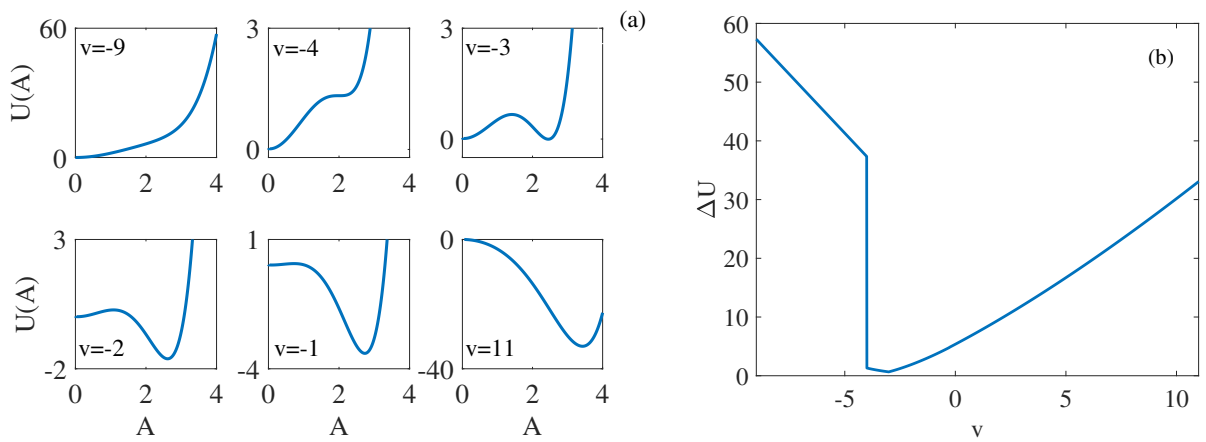

Figure 15: Influences of the parameter $v$ on potential function. (a) Variation of potential function $U(A)$ under different parameter $v$. (b) Relationship between barrier height $\Delta U$ and parameters $v$.

we consider that the weight of rate in the factors that promote tipping is a fixed value, the greater the total difficulty of tipping, the larger contribution of the rate is needed, and the the effect difference between different rates. It is like when we are climbing a 2000-meter mountain, if we use crutches and other equipment, we will feel that the effect of assistance is obvious. However, if the mountain we climb is only 500 meters, the presence or absence of crutches seems to have little effect on the climbing speed, and the quality of crutches is not so important. From the perspective of barrier height difference, the difficulty of tipping when the parameter rises is greater than that when the parameter falls, so the size of rate has a significant effect on tipping-delay when the parameter rises, while the size of rate has little effect on tipping-delay when the parameter falls. As generalisation of Gaussian white noise, the result in Lévy noise has similar mechanism and phenomenon.

\subsection{Early warning signals for Lévy rate-dependent tipping system}

This section considers the early warning signals of rate-dependent thermoacoustic system excited by Lévy noise. Figure 16 shows the variation of the amplitude $A$, lag-1 autocorrelation (AC), variance (VAR), skewness (SKEW), kurtosis (K), and entropy (Entropy) during tipping, see the Appendix for the definitions. The red dashed line in each subfigure represents the maximum value of amplitude change rate, which is the timming index of tipping. As can be seen from Figure 16(a), the location of timing index of tipping is about in the middle of the transition process from low amplitude to high amplitude. The lag-1 autocorrelation and variance plotted in Figures 16(b) and (c) are commonly used early warning indicators in B-tipping, which are based on the theory of critical slowing down. The skewness and kurtosis shown in Figures 16(d) and (e) are indicators to describe the probability density. The skewness is a measure of the asymmetry of the probability density, and the kurtosis reflects the shape of the probability density peak. The entropy calculated in Figure 16(f) is 
an indicator reflecting the degree of confusion in the system, and it has not been considered as an early warning signal in the rate-dependent system before.
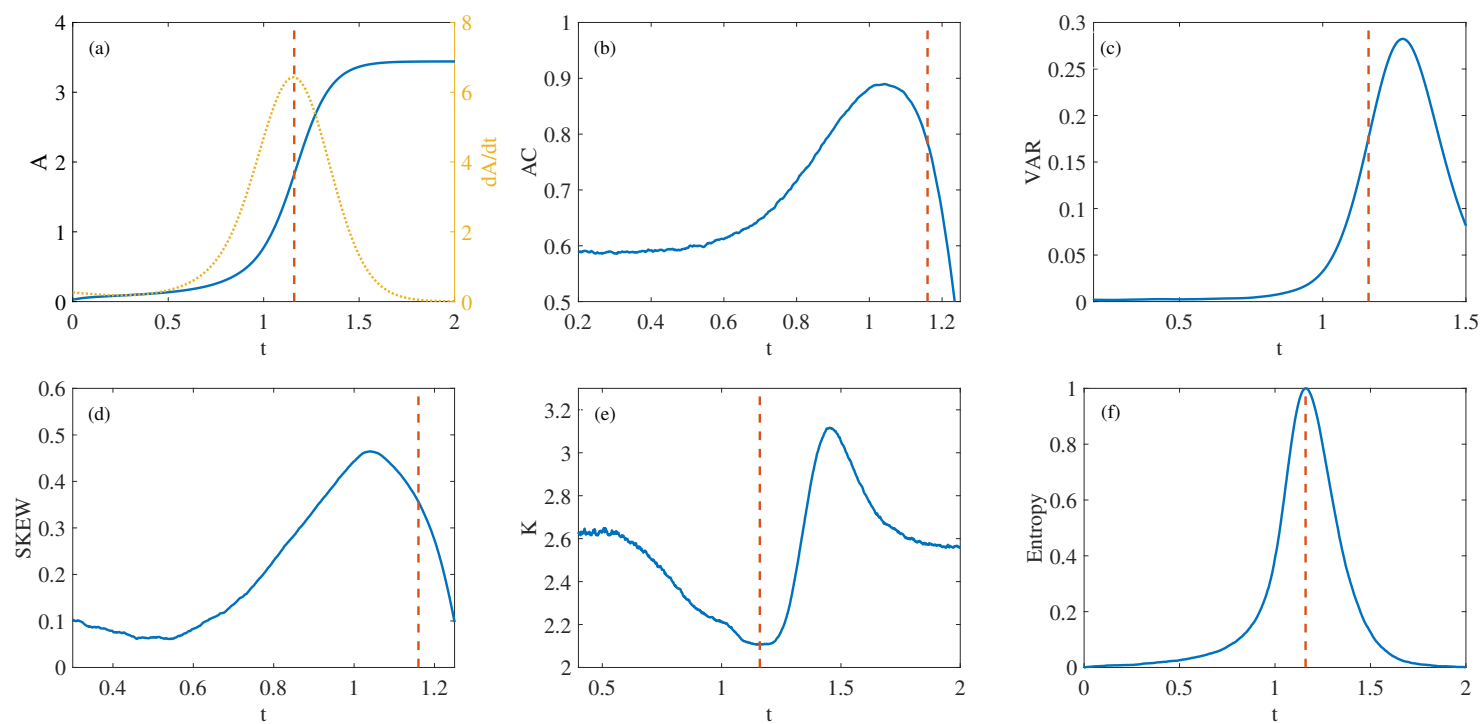

Figure 16: Variations of (a) amplitude $A$, (b) lag-1 autocorrelation, (c) variance, (d) skewness, (e) kurtosis, and (f) entropy with time. The point of the maximum rate of change of $A$ is marked by a red dashed line.

From Figure 16, the highest values of variance and kurtosis are after the timing index of tipping, which cannot be used as early warning signals under the current system. The highest values of lag-1 autocorrelation, skewness and entropy all appear no later than the timing index of tipping, and they all suddenly increase before tipping, so they can make predictions about tipping. The highest values of lag-1 autocorrelation and skewness under different parameters cannot be determined. We can only predict tipping when they reach the highest point. However, the highest point of entropy must be 1, and it is near the occurrence of tipping. In this way, we can judge how long before the tipping will take place by the difference between the current entropy value and the maximum value of 1 . Compared with lag-1 autocorrelation and skewness, entropy has more maneuverability in controlling the system.

Next, the warning effect of entropy on rate-dependent system is considered in both time space and parameter space. Figures $17(\mathrm{a}-\mathrm{e})$ show the variation of entropy with time under different rates $R$ in time space. Take Entropy $=0.05$ as the threshold for example (the yellow dotted line in the Figure 17), then make the difference between the time when the entropy first passes the threshold and the actual time when tipping occurs (the maximum value of amplitude change rate), and the difference is regarded as the warning time (the blue shaded part in the Figure 17). We find that the larger the rate $R$ is, the earlier the tipping 
occurs and the shorter the warning time is.
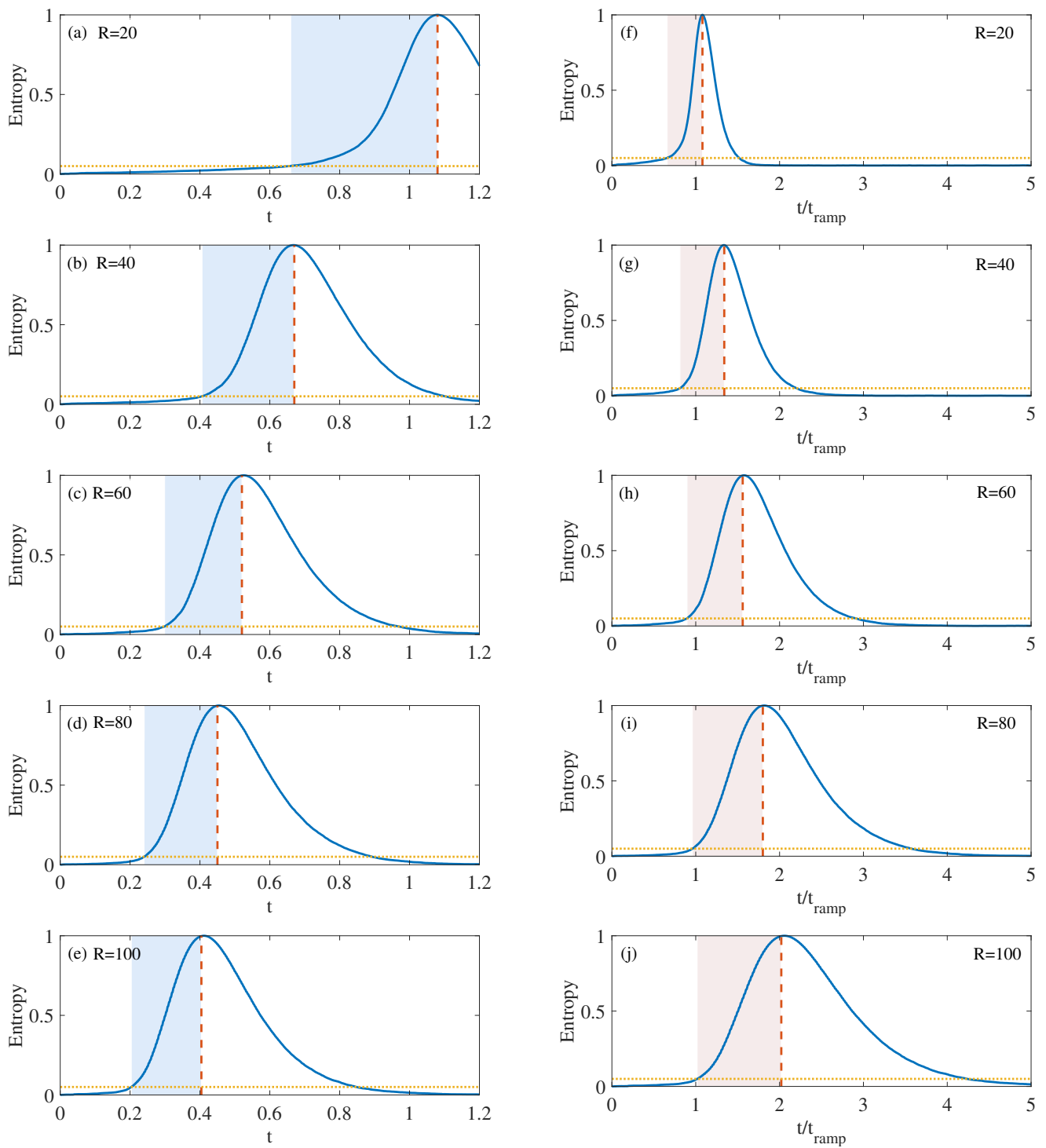

Figure 17: Early warning of (a-e) entropy under different rates $R$ in time space. (f-j) entropy under different rates $R$ in parameter space.

The abscissa of Figures 17(f-j) unifies the time scale, and the actual consideration is the change of entropy in the parameter space. Similar to the definition in time space, we can also define the warning of parameter interval in parameter space (the red shaded part in the Figure 17), that is, the difference between the abscissa value of first passing the threshold of 0.05 and the abscissa of actual tipping. It is found that the larger the rate $R$ is, the larger the warning of parameter range is, and better the effect is. In conclusion, the early warning effect of parameter change rate $R$ on time space and parameter space is different. So we should adjust the change rate of parameters and make the control strategy according to the 
actual demand of time space and parameter space.

\section{Conclusions}

In this work, we investigate the dynamical behaviours of rate-dependent thermoacoustic systems whose parameters vary with time. We find that systems with subcritical Hopf bifurcation excited by non-Gaussian Lévy noise are more likely to cause tipping than Gaussian noise, and the delay time of tipping-delay phenomenon is shorter. This indicates that thermoacoustic instabilities are more likely to occur and more difficult to control for thermoacoustic systems in extreme working environments modelled by non-Gaussian Lévy noise. To this end, we have considered a two-pronged analysis. On the one hand, after our analysis of several important parameters such as the stability index, the skewness index, and the noise intensity of Lévy noise, as well as considering the coupling effect of the parameter change rate, we come up with several control strategies. The occurrence of thermoacoustic instability can be circumvented by increasing the rate of change of the parameter $R$, increasing the stability index $\alpha$ and reducing the noise intensity $D$ to delay the onset of tipping. On the other hand, the tipping points are predicted by early warning signals so that other control measures can be taken. It is verified that the lag-1 autocorrelation, the skewness and the entropy can be used as early warning signals. More appropriately, entropy is a better indicator in comparison with other traditional ones. Entropy has more operability in taking control of the system, and its early warning effect is also related to the rate of parameter change.

Our results have important implications for research in the development of power propulsion devices such as rocket engines, gas turbines, and space engines. As research continues, the extreme operating environment of the engine is a factor that cannot be ignored and can make the systems already prone to thermoacoustic instability even worse. And our investigation on the rate-dependent thermoacoustic system with non-Gaussian Lévy noise provides theoretical guidance for engine design and effective ideas for subsequent control, which is also enlightening for the research in related fields.

\section{Acknowledgments}

This work was partly supported by the NSF of China (12072264), the Key International (Regional) Cooperative Research Projects of the NSF of China (12120101002), the Research Funds for Interdisciplinary Subject of NPU, the Shaanxi Provincial Key R\&D Program (Grants 2020KW-013, 2019TD-010). 


\section{Appendix: Basic concepts of early warning measures}

In this appendix, we provide some basics definitions used for calculating early warning signals.

\subsection{Variance}

Variance (VAR) is a measure of dispersion in probability theory and statistical variance when measuring a random variable or a set of data. It is defined as

$$
\mathrm{VAR}=\frac{1}{N} \sum_{i=1}^{N}[x(i)-\mu]^{2},
$$

where $\mu=\frac{1}{N} \sum_{i=1}^{N} x(i)$ is the mean and $N$ is the number of data points.

\subsection{Autocorrelation}

Autocorrelation (AC), is the correlation of a signal with itself at different points in time. Informally, it is the similarity between two observations as a function of the time difference between them. It is a mathematical tool to find repeating patterns or to identify fundamental frequencies that disappear implicitly in the harmonic frequencies of a signal. The autocorrelation can be expressed as a function of time delay $\tau$,

$$
\mathrm{AC}(\tau)=\frac{1}{(N-\tau) \cdot \operatorname{VAR}} \sum_{i=1}^{N-\tau}[x(i)-\mu][x(i+1)-\mu] .
$$

In this paper we use the case when $\tau=1$, i.e., lag-1 autocorrelation.

\subsection{Skewness}

Skewness (SKEW) is a measure of the asymmetry of the probability density. If the skewness is equal to zero, the probability density is symmetric about the mean. If the skewness is negative, the probability density is skewed to the left and to the right for positive values. It is defined as the third order moment,

$$
\mathrm{SKEW}=\frac{1}{N \cdot(\mathrm{VAR})^{3 / 2}} \sum_{i=1}^{N}[x(i)-\mu]^{3} .
$$

\subsection{Kurtosis}

Kurtosis $(\mathrm{K})$ reflects the shape of the probability density peak. The larger value corresponds to a sharper peak. It is defined as the fourth order moment,

$$
\mathrm{K}=\frac{1}{N \cdot(\mathrm{VAR})^{2}} \sum_{i=1}^{N}[x(i)-\mu]^{4} .
$$




\subsection{Entropy}

Entropy is an effective means to quantify the dynamical characteristics of a system, and also to react to qualitative changes in the dynamical behaviour of the system through its changes. If the possible states of the random variable $X$ are $X_{1}, X_{2}, \ldots, X_{n}$, and the corresponding probability of each state occurring is $P(X i), i=1,2, \ldots, n$. Then, the entropy of the random variable $X$ is defined as

$$
\text { Entropy }=-\sum_{i=1}^{n} P\left(X_{i}\right) \log P\left(X_{i}\right) .
$$

\section{Data availability}

The data that supports the findings of this study are available within the article.

\section{References}

[1] A. A. Putnam, W. R. Dennis, Survey of organ-pipe oscillations in combustion systems, The Journal of the Acoustical Society of America 28 (2) (1956) 246-259.

[2] K. T. Feldman Jr, Review of the literature on Sondhauss thermoacoustic phenomena, Journal of Sound and Vibration 7 (1) (1968) 71-82.

[3] C. Sondhauss, Ueber die Schallschwingungen der Luft in erhitzten Glasröhren und in gedeckten Pfeifen von ungleicher weite, Annalen der Physik 155 (1) (1850) 1-34.

[4] T. C. Lieuwen, Unsteady Combustor Physics, Cambridge University Press, 2012.

[5] P. D. Ritchie, J. J. Clarke, P. M. Cox, C. Huntingford, Overshooting tipping point thresholds in a changing climate, Nature 592 (7855) (2021) 517-523.

[6] P. Ashwin, S. Wieczorek, R. Vitolo, P. Cox, Tipping points in open systems: bifurcation, noise-induced and rate-dependent examples in the climate system, Philosophical Transactions of the Royal Society A 370 (1962) (2012) 1166-1184.

[7] Y. Malhi, L. E. Aragão, D. Galbraith, C. Huntingford, R. Fisher, P. Zelazowski, S. Sitch, C. McSweeney, P. Meir, Exploring the likelihood and mechanism of a climate-changeinduced dieback of the amazon rainforest, Proceedings of the National Academy of Sciences 106 (49) (2009) 20610-20615.

[8] P. D. Ditlevsen, S. J. Johnsen, Tipping points: Early warning and wishful thinking, Geophysical Research Letters 37 (19) (2010). 
[9] J. Lohmann, P. D. Ditlevsen, Risk of tipping the overturning circulation due to increasing rates of ice melt, Proceedings of the National Academy of Sciences 118 (9) (2021).

[10] G. Bonciolini, D. Ebi, E. Boujo, N. Noiray, Experiments and modelling of ratedependent transition delay in a stochastic subcritical bifurcation, Royal Society Open Science 5 (3) (2018) 172078.

[11] G. Bonciolini, N. Noiray, Bifurcation dodge: avoidance of a thermoacoustic instability under transient operation, Nonlinear Dynamics 96 (1) (2019) 703-716.

[12] X. Zhang, Y. Xu, Q. Liu, J. Kurths, Rate-dependent tipping-delay phenomenon in a thermoacoustic system with colored noise, Science China-Technological Sciences 63 (11) (2020) 2315-2327.

[13] X. Zhang, Y. Xu, Q. Liu, J. Kurths, C. Grebogi, Rate-dependent bifurcation dodging in a thermoacoustic system driven by colored noise, Nonlinear Dynamics 104 (3) (2021) $2733-2743$.

[14] Y. M. Blanter, M. Büttiker, Shot noise in mesoscopic conductors, Physics Reports $336(1-2)(2000) 1-166$.

[15] K. Kanazawa, T. Sagawa, H. Hayakawa, Stochastic energetics for non-gaussian processes, Physical Review Letters 108 (21) (2012) 210601.

[16] W. Schoutens, Lévy Processes in Finance: Pricing Financial Derivatives, Wiley Online Library, 2003.

[17] X. Zhang, Y. Xu, B. Schmalfuß, B. Pei, Random attractors for stochastic differential equations driven by two-sided Lévy processes, Stochastic Analysis and Applications 37 (6) (2019) 1028-1041.

[18] P. D. Ditlevsen, Observation of $\alpha$-stable noise induced millennial climate changes from an ice-core record, Geophysical Research Letters 26 (10) (1999) 1441-1444.

[19] X. Shen, H. Zhang, Y. Xu, S. Meng, Observation of alpha-stable noise in the laser gyroscope data, IEEE Sensors Journal 16 (7) (2015) 1998-2003.

[20] A. Corral, Universal earthquake-occurrence jumps, correlations with time, and anomalous diffusion, Physical Review Letters 97 (17) (2006) 178501. 
[21] M. Scheffer, J. Bascompte, W. A. Brock, V. Brovkin, S. R. Carpenter, V. Dakos, H. Held, E. H. Van Nes, M. Rietkerk, G. Sugihara, Early-warning signals for critical transitions, Nature 461 (7260) (2009) 53-59.

[22] J. Ma, Y. Xu, Y. Li, R. Tian, J. Kurths, Predicting noise-induced critical transitions in bistable systems, Chaos 29 (8) (2019) 081102.

[23] J. Ma, Y. Xu, W. Xu, Y. Li, J. Kurths, Slowing down critical transitions via Gaussian white noise and periodic force, Science China-Technological Sciences 62 (12) (2019) $2144-2152$.

[24] I. Pavithran, R. Sujith, Effect of rate of change of parameter on early warning signals for critical transitions, Chaos 31 (1) (2021) 013116.

[25] D. Applebaum, Lévy Processes and Stochastic Calculus, Cambridge university press, 2009. 